Bull. Mater. Sci., Vol. 22, No. 6, October 1999, pp. 947-951. (C) Indian Academy of Sciences.

\title{
Transmission electron microscopy and X-ray diffraction studies of quantum wells
}

\author{
D V SRIDHARA RAO ${ }^{\dagger}$, K MURALEEDHARAN ${ }^{\dagger}$, G K DEY ${ }^{\ddagger}$, S K HALDER ${ }^{\S}$, \\ G BHAGAVANNARAYAN ${ }^{\S}$, P BANERJI, D PAL and D N BOSE* \\ Advanced Technology Centre, Indian Institute of Technology, Kharagpur 721 302, India \\ ${ }^{\dagger}$ Electron Microscopy Group, Defence Metallurgical Research Laboratory, Hyderabad 500058 , India \\ ${ }^{\ddagger}$ Materials Science Division, Bhabha Atomic Research Centre, Mumbai 400 085, India \\ ${ }^{\S}$ Materials Characteristics Division, National Physical Laboratory, New Delhi 110012 , India
}

MS received 22 July 1999

\begin{abstract}
A series of $\operatorname{In}_{x} \mathrm{Ga}_{1-x} \mathrm{As}(x=0 \cdot 47)$ quantum wells with InP barrier layers have been grown on InP substrates by metalorganic vapour phase epitaxy (MOVPE) at $625^{\circ} \mathrm{C}$. The nominal well widths were defined during growth at (i) $25 \AA, 39 \AA, 78 \AA$ and $150 \AA$ for one sample and (ii) $78 \AA$ for all 4 wells in another sample. The InP barrier widths have been kept constant at $150 \AA$. These layers have been characterized by $X$-ray diffraction (XRD) which from simulation gave the nominally $78 \AA$ well width as $84 \AA$ and the nominally $150 \AA$ barrier width as $150.5 \AA$. Transmission electron microscopy (TEM) and high resolution TEM (HRTEM) have been carried out on etched and ion-milled samples for direct measurement of well and barrier widths. The well widths found from TEM are $25 \AA, 40 \AA, 75 \AA$ and $150 \AA$. TEM micrographs revealed that, while the InP barrier layer is of good quality and the growth is confirmed to be epitaxial, dipoles are detected at the interface and the quantum well has some small disordered regions. These thickness measurements are in good agreement with earlier photoluminescence (PL) and secondary.ion mass spectrometry (SIMS) studies.
\end{abstract}

Keywords. Quantum wells; MOVPE growth; X-ray diffraction; TEM.

\section{Introduction}

The characterization of quantum wells has been carried out using the entire gamut of techniques available to materials scientists, some of these being stretched to their limits. The parameters of interest are morphology, layer thickness, composition, crystallinity, interface and defect structure. The most widely used techniques for structural characterization have been X-ray diffraction (XRD) and double crystal X-ray diffraction (DXRD) from which it is possible to determine the layer thicknesses, lattice mismatch and hence the composition (Razeghi 1989). The width of the rocking curve also gives an estimate of crystalline quality. Transmission electron microscopy is a powerful technique which has been shown to yield extremely precise information on a microscopic scale (Petroff et al 1978) and from which the layer thickness can be found directly. High resolution TEM (HRTEM) gives lattice imaging on the atomic scale which shows up structural defects at the interface. Ourmazd et al (1986) have described the fundamentals of HRTEM imaging of III-V compounds and shown how chemical information i.e. identification of the sublattices can be obtained using a $400 \mathrm{kV}$ JEOL model 4000-EX HRTEM. The difficulties

*Author for correspondence in specimen preparation which includes ion milling of delicate and brittle semiconductor samples have hitherto limited the use of this technique in India. We present the first work in which the entire growth and characterization of $\mathrm{InGaAs} / \mathrm{InP}$ quantum wells was carried out in various laboratories within the country.

All the early work on quantum wells was carried out on the GaAs/AlGaAs system (Petroff et al 1978) which was the workhorse of growth by molecular beam epitaxy (MBE) and metalorganic vapour phase epitaxy (MOVPE). This system is naturally lattice-matched and provided the first quantum well devices. Since then much work has focussed on the $\operatorname{InP} / \mathrm{In}_{x} \mathrm{Ga}_{1-x} \mathrm{As}$ and $\operatorname{InP} / \mathrm{In}_{x} \mathrm{Ga}_{1-x} \mathrm{As}_{y} \mathrm{P}_{1-y}$ systems because of their importance in fibre-optic communication at $1.3 \mu \mathrm{m}$ and $1.55 \mu \mathrm{m}$ wavelengths. We have reported MOVPE growth and characterization of $\operatorname{In}_{x} \mathrm{Ga}_{1-x} \mathrm{As} / \mathrm{InP}$ quantum wells using photoluminescence (PL) (Bose et al 1998) and secondary ion mass spectrometry (SIMS) (Bose et al 1999) techniques. In this short report we present DXRD, TEM and HRTEM characterization of these layers which testify to their high quality.

\section{Growth}

$\mathrm{In}_{x} \mathrm{Ga}_{1-x} \mathrm{As}$ quantum wells with InP barrier layers on (100-2 ${ }^{\circ}$ off towards 110) InP substrates were grown by atmospheric pressure MOVPE using a Thomas Swan 
system. This was fully computer-controlled with a fast switching manifold for growth of thin multilayers. The use of the alkyls as metal sources results in the reduction of growth temperatures below those required for conventional CVD, the other reactants being $\mathrm{AsH}_{3}$ and $\mathrm{PH}_{3}$. Growth is carried out using ultra high-purity hydrogen, obtained from a Pd-diffuser, as carrier gas. The growth

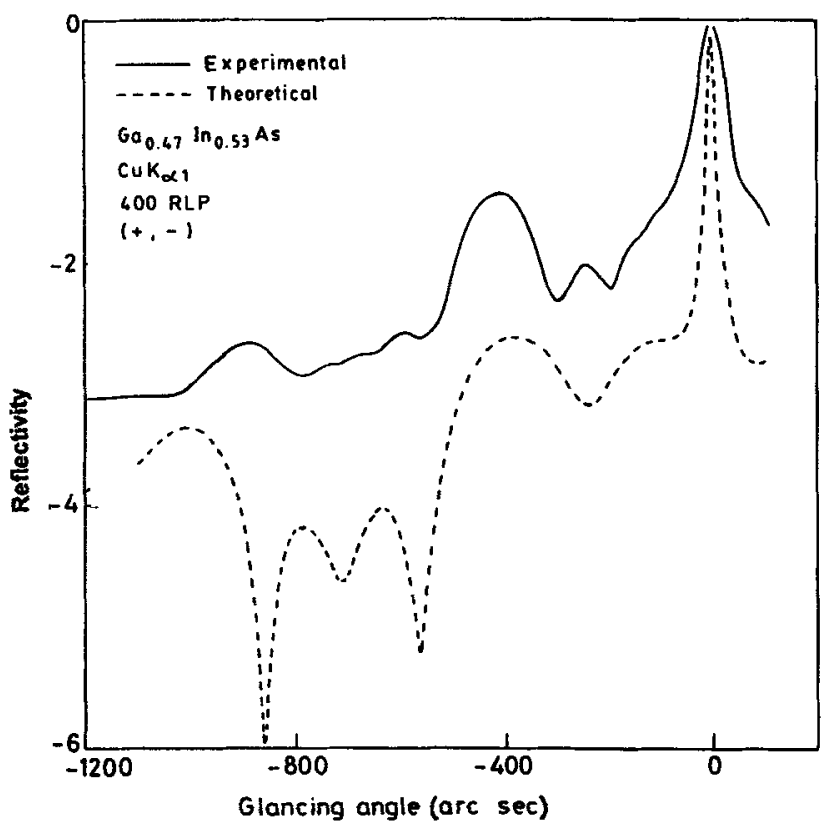

Figure 1. X-ray reflectivity curve for a InGaAs/InP MQW structure giving theoretical and experimental plots (well $=78 \AA$, barrier $=150 \AA$ ). temperature was optimized at $625^{\circ} \mathrm{C}$ using PL and SIMS as monitors, to yield emission with lowest linewidth and layers with sharpest -interfaces. The lattice-matched composition $\operatorname{In}_{x} \mathrm{Ga}_{1-x}$ As with $x=0.47$, confirmed from DXRD, was obtained by varying the tri-methyl indium (TMI) and tri-methyl gallium (TMG) flow rates which were kept at 120 and $6.3 \mathrm{sccm}$, respectively. In the first set of growth runs 4 wells of different thicknesses viz. $25 \AA, 39 \AA, 78 \AA$ and $150 \AA$ were grown and the widths estimated from their PL signatures. The $39 \AA$ well gave a narrow PL linewidth as expected while the $25 \AA$ well gave considerable linewidth broadening due to variation of well width. The InP barriers were kept constant at $300 \AA$. It was found that $78 \AA$ wells gave emission at $1.55 \mu \mathrm{m}$ as required for fibre-optic laser sources. Hence for the actual lasers 4 equal wells of $78 \AA$ thickness were grown with $150 \AA$ InP barriers. The growth rate of the InP layer was estimated to be $\sim 8.25 \AA / s$ under the experimental conditions, which can be compared with a monolayer thickness of $5.69 \AA$. Comparison between PL and SIMS studies showed the presence of interfacial layers between well and barrier of monolayer width due to the finite switching time $\sim 1 \mathrm{~s}$ and also due to remanent gas effects in the reactor. Characterization by DXRD, TEM and HRTEM are reported here, the latter throwing light on the nature of interfacial defects.

\section{X-ray diffraction}

$\mathrm{X}$-ray diffraction studies on $\mathrm{In}_{x} \mathrm{Ga}_{1-x} \mathrm{As} / \mathrm{InP}$ multiquantum wells (MQW) grown by MOVPE were reported by Razeghi (1989) on a 10-period superlattice with

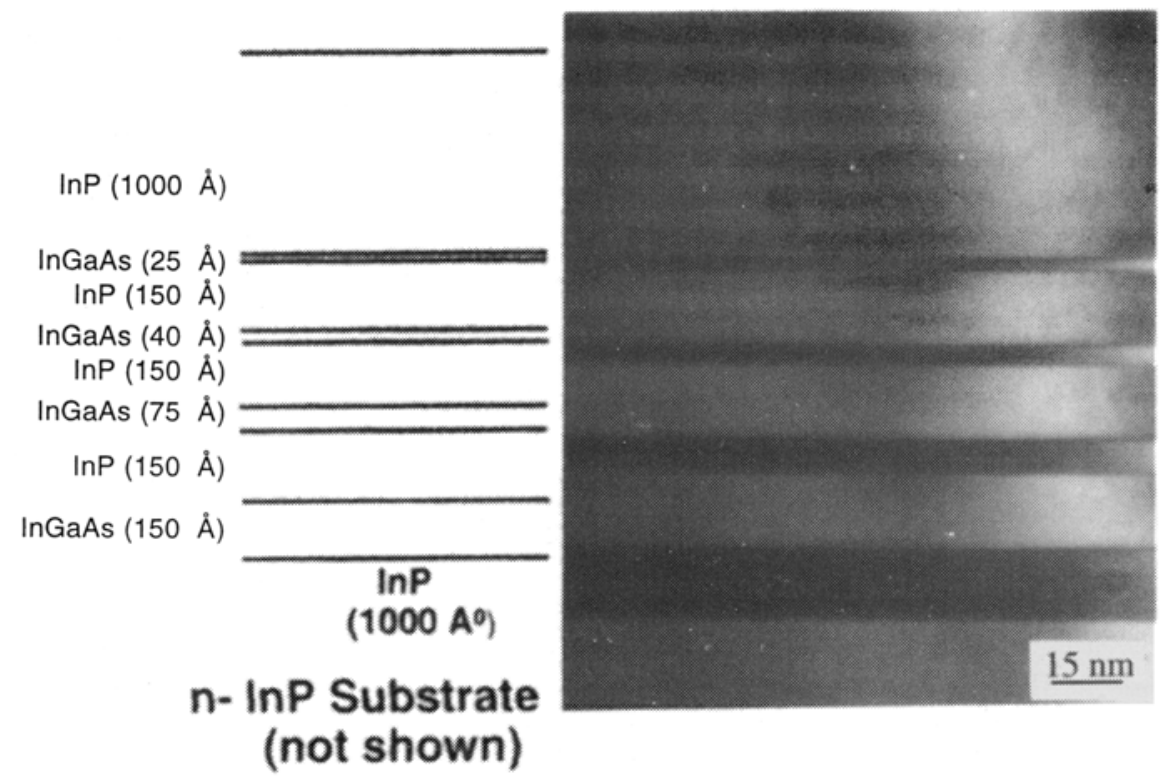

Figure 2. Transmission electron micrograph of quantum wells of nominal widths $25 \AA$, $39 \AA, 78 \AA, 150 \AA$; barrier width $300 \AA$. 
$w=200 \AA$ and $b=250 \AA$. In this case a synchrotron radiation source was used. The $(400)$ reflection gave satellite peaks up to $n=5$, from which lattice periodicity was determined to an accuracy of $\pm 20 \AA$.

In the present case $\mathrm{X}$-ray diffraction studies were carried out using a commercial Rigaku double crystal Xray diffractometer with rotating anode source using $\mathrm{CuK}_{\alpha 1}$ $(\lambda=1.54 \AA)$ radiation in $(+,-)$ configuration. The thicknesses of the quantum well and the barrier as well as the composition of the quantum well have been determined by the simulation of the experimentally recorded rocking curve on the basis of the semi-kinematical theory given by Kyutt et al (1980) for characterization of ion-implanted samples. The detailed description of the theory for the quantum wells will be published elsewhere. As seen from figure 1 the fit between experiment and theory is reasonably good, having well resolved oscillations due to the quantum wells and barriers. It may be mentioned that

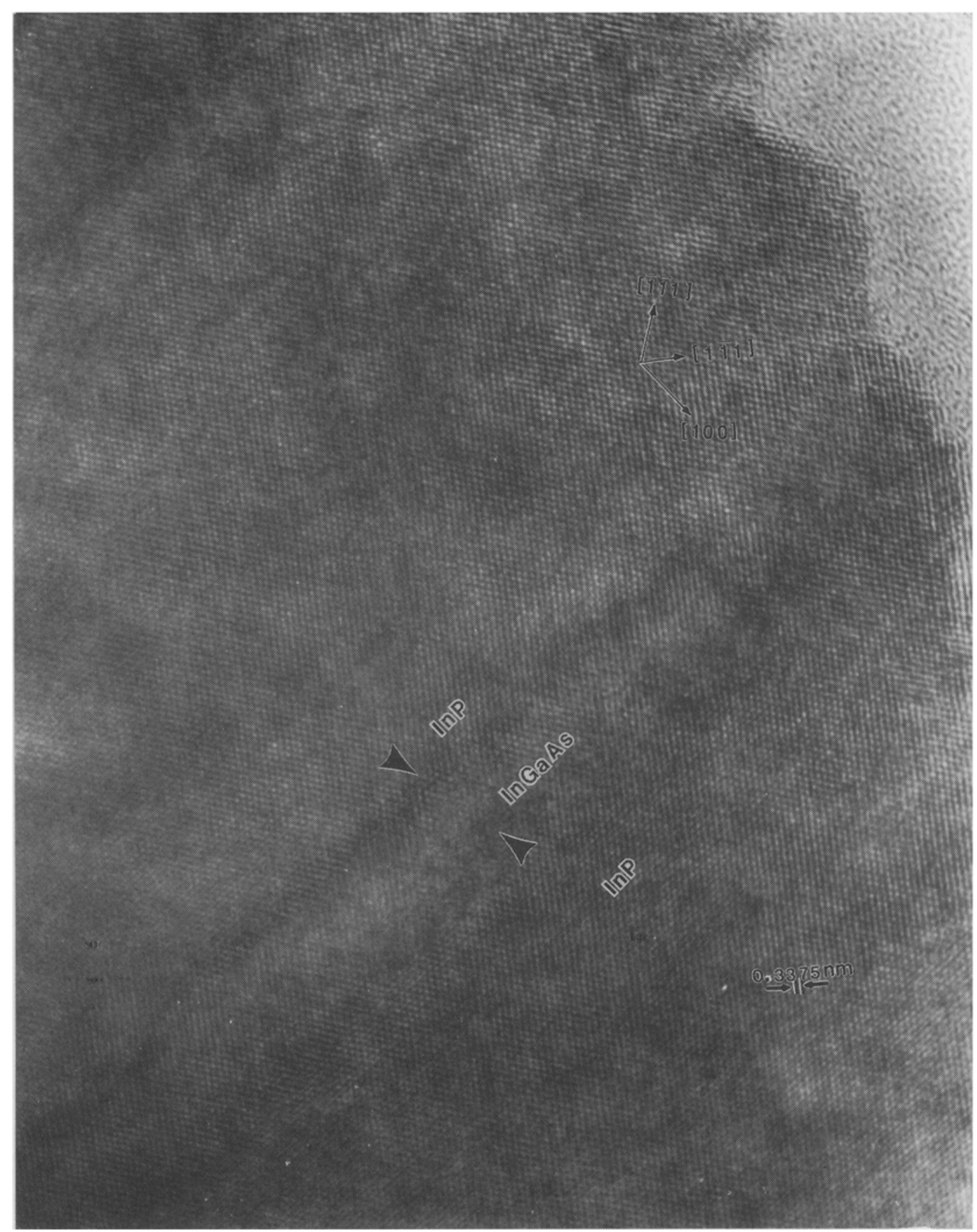

Figure 3. HRTEM of InGaAs quantum well between two InP barrier layers. 
the $y$-axis of the experimental curve is in arbitrary units and has insignificant effect on the simulation. The broadness of the experimental peak in comparison with the dynamical peak is as expected even in the case of a virgin substrate. From the simulation, the best fit values obtained are as follows: (i) composition $x$ of $\mathrm{In}_{x} \mathrm{Ga}_{1-\mathrm{x}} \mathrm{As}$ quantum well is 0.47 , (ii) quantum well width, $w=84 \AA$ and barrier width, $b=150.5 \AA$ and (iii) number of quantum wells $=4$. The error in the value of $x$ is within $\pm 0.5 \%$. The error values in the thicknesses of the barrier width and well width are $1 \%$ and $5 \%$, respectively. The simulated values are close to the values expected from the growth parameters which are $w=78 \AA, b=150 \AA$ and $n=4$.

\section{Transmission electron microscopy}

A 40-period $\operatorname{In}_{x} \mathrm{Ga}_{1-x} \mathrm{As} / \mathrm{InP}$ superlattice grown by MOCVD was examined by TEM by Razeghi et al (1989). For the layer grown with computer control, as in the present case, the thickness homogeneity was found to be excellent. Dark field images with atomic resolution were also obtained. Atomic steps every $80 \AA$ could be seen due to the $2^{\circ}$ mis-orientation of the InP substrate.

For the present experiments a cross-sectional TEM foil of the MQW structure was first prepared by making a sandwich structure with two pieces of the sample glued by a high temperature fast-curing epoxy (Devcon 5 min epoxy) so that the multilayers are face-to-face. The dimensions of the sandwich structure are $0.35 \mathrm{~mm} \times 2.2 \mathrm{~mm} \times 10 \mathrm{~mm}$. This structure is fixed in a centrally slotted molybdenum wire having $2.6 \mathrm{~mm}$ diameter, which in turn is fixed, using the same epoxy, in a brass tube having $2.7 \mathrm{~mm}$ internal and $3.0 \mathrm{~mm}$ external diameter. Thin foils of thickness about $200 \mu$ were cut from this by a slow speed diamond saw. One of the foils was selected for studies. This foil was mechanically thinned to about $100 \mu$ by lapping, followed by dimpling on both sides by a Gatan dimpler (Model 656). The final thickness of the foil in the central region was about $30 \mu$. Then the foil was ion milled by Iolar-2 grade high purity argon with $5 \mathrm{kV}$ gun voltage, $12^{\circ}$ gun angle and $75 \mu \mathrm{A}$ specimen current (Gatan duo mill model 600) for electron transparency thickness, employing a liquid nitrogen cold stage.

TEM studies on this cross-sectional foil were carried out by Philips EM $430 \mathrm{~T}$ analytical TEM $(200 \mathrm{kV})$ and JEOL 3010 High Resolution TEM $(300 \mathrm{kV})$. Figure 2 shows a bright field TEM micrograph showing the crosssection of a MQW structure with four $\operatorname{In}_{x} \mathrm{Ga}_{1-x}$ As quantum wells of nominal widths $150 \AA, 78 \AA, 39 \AA$ and $25 \AA$, in order of growth on the substrate, the interfaces being on an edge-on orientation. The well thicknesses as measured from TEM are: $150 \AA, 75 \AA, 40 \AA$ and $25 \AA$ and the barrier widths $150 \AA$ in good agreement with the experimental growth parameters. Figure 3 is a high resolution micrograph of the $\mathrm{In}_{x} \mathrm{Ga}_{1-x} \mathrm{As}(40 \AA)$ quantum well sandwiched between two InP barrier layers. An analysis of this image shows that the overall microstructure is good, especially that the InP barrier layers are

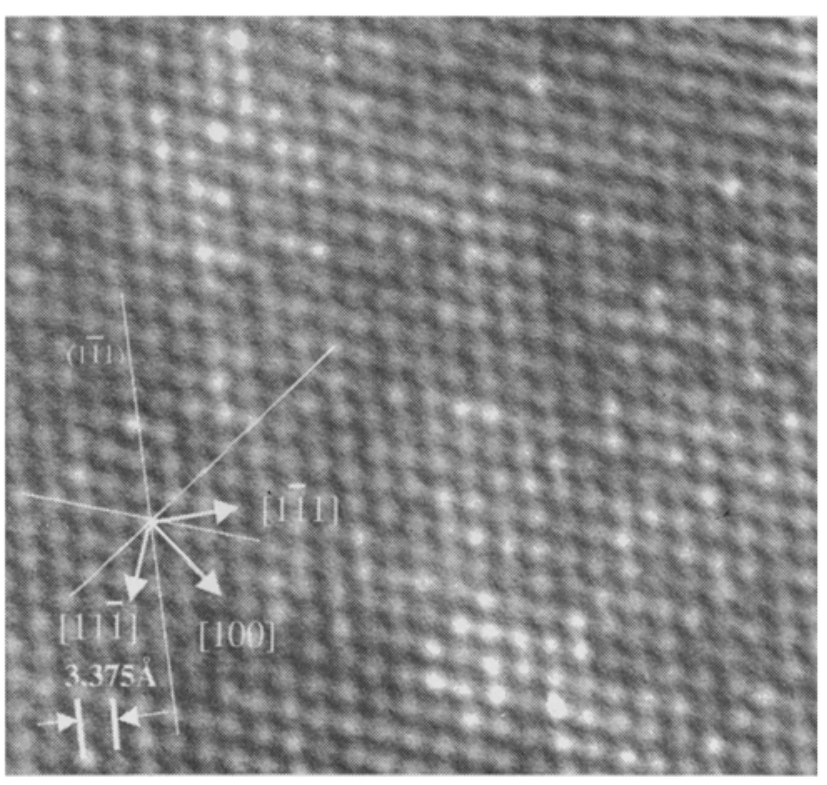

Figure 4. HRTEM of InP barrier layers showing different lattice directions.

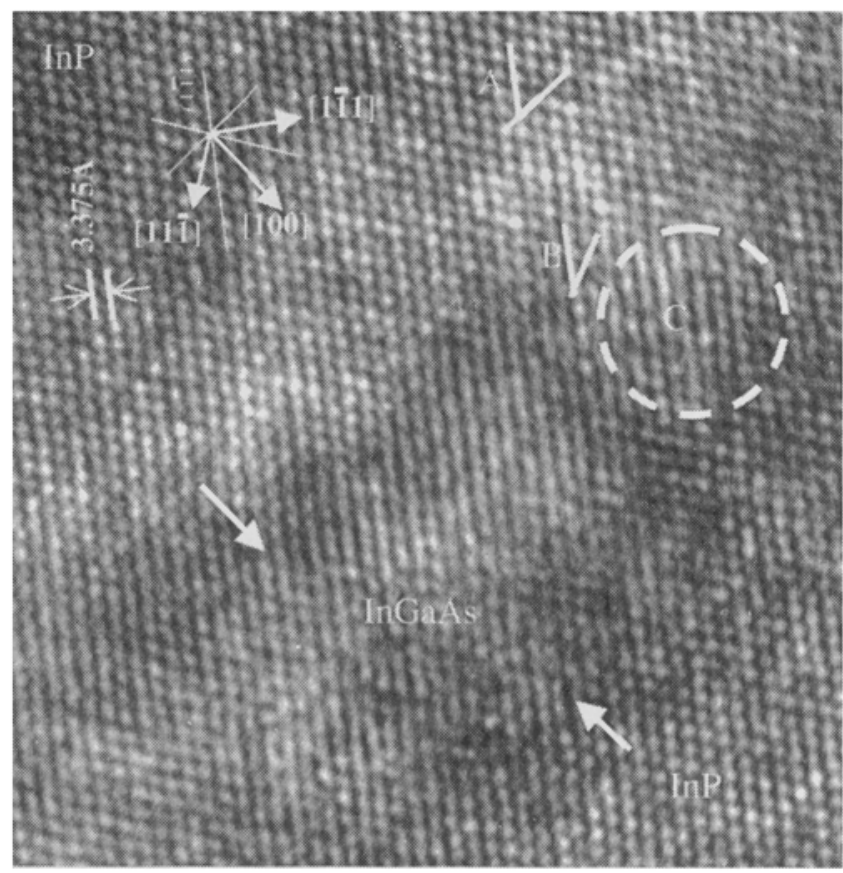

Figure 5. HRTEM of InGaAs well showing different lattice planes and defects. 


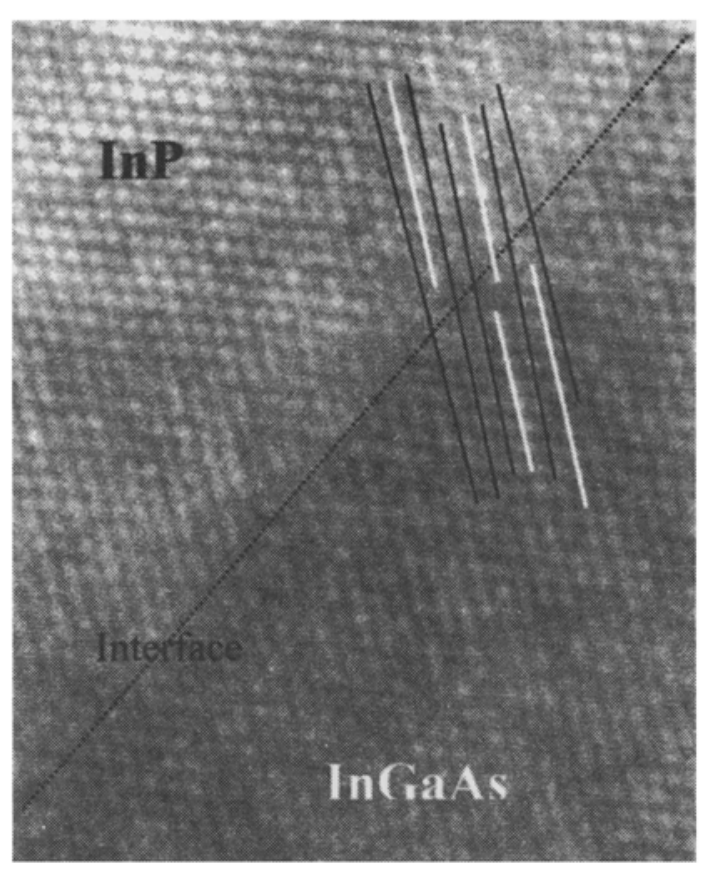

Figure 6. HRTEM of InGaAs/InP interface showing the presence of dipoles.

grown with minimum microdefects, as shown in figure 4 . The growth process has induced a certain amount of strain in the epilayer, as one can see the strain contrast across the interface, as shown by arrows near the interface. It is noticed from figure 5 that few localized microstructural imperfections are incorporated in the epilayers as one can see the slight distortion of atomic arrangement near the interface, as the angles between the (100) and (111) planes are varying in these regions: one such region is shown as region $\mathrm{B}$, while region $\mathrm{A}$ shows an undistorted area. It is noticed that the atomic positions could not be located precisely in certain regions, specially in the interface region shown as $\mathrm{C}$. Across the interface between $78 \AA \operatorname{In}_{x} \mathrm{Ga}_{1-x} \mathrm{As}$ epilayer and the InP epilayer, dipoles are noticed in a few pockets, as shown in figure 6. Although the global microstructure looks good, it can be further improved by avoiding these localized imperfections by fine-tuning the growth conditions.

\section{Conclusions}

Quantum wells and ordered superlattices form the basis of new families of optoelectronic devices such as lasers, LEDs and infra-red detectors (QWIPs). It is important to be able to grow and characterize them in detail using modern atomic-level tools. The present study has focussed on lattice-matched system $\mathrm{In}_{x} \mathrm{Ga}_{1-x} \mathrm{As} / \mathrm{InP}$ which is important in fibre-optics. While $\mathrm{X}$-ray diffraction is one of the most widely used techniques, TEM has been shown to provide valuable information not obtainable otherwise. Good agreement has been found in assessing the dimensions and overall quality of MOCVD-grown quantum wells by these two techniques.

\section{Acknowledgements}

The authors are grateful to $\mathrm{Dr} A \mathrm{~K}$ Raychoudhuri, Director, National Physical Laboratory, New Delhi, Dr D Banerjee, Director, Defence Metallurgical Research Laboratory and Dr S Banerjee, Head, Materials Science Division, Bhabha Atomic Research Centre, for their support in carrying out this work. The authors are grateful to Dr D D Bhawalkar, Chairman, National Laser Programme, for sustained funding of the project on 'Semiconductor Lasers'.

\section{References}

Bose D N, Banerji P and Pal D 1998 Proc. Photonics-98 (New Delhi: Viva books) p 551

Bose D N, Banerji P, Bhunia S, Aparna Y, Chetri M B and Chakrabarti B R 1999 Appl. Surf. Sci. (communicated)

Kyutt R N, Petrashen P V and Sorokin L M 1980 Phys. Status Solidi (a)60 381

Ourmazd A, Rentschler J R and Taylor D W 1986 Phys. Rev. Lett. 573073

Petroff P M, Gossard A C, Wiegmann W and Savage A 1978 J. Cryst. Growth 445

Razeghi M 1989 The MOCVD challenge (Bristol: Adam Hilger) Vol. 1 